\title{
A Simulation Study on Triple Frequency Ambiguity Resolution for Reference Stations Using Different Strategy Regarding Elevation angles
}

\author{
Sun-Kyoung $\mathrm{Yu}^{1}$, Dong-uk Kim, June-sol Song ${ }^{2}$, and Changdon $\mathrm{Kee}^{1^{*}}$ \\ ${ }^{1}$ School of Mechanical and Aerospace Engineering and the SNU-IAMD, 08826 Seoul, Korea \\ ${ }^{2}$ ENAC, France
}

\begin{abstract}
This paper proposes an ambiguity resolution method using triple frequency for reference stations. Using the reference coordinate information, geometry based ambiguity resolution performance is analysed. Although orbit errors and tropospheric model errors still remain, wide lane ambiguity could be fixed in several epochs. However, the narrow lane wave length of about $10 \mathrm{~cm}$ is too short to overcome error sources by simply combining the measurement. Therefore, we have divided the elevation angle into 5 degree intervals and investigated the measurement errors and the time to fix of each section. For high elevation satellites, it is possible to determine in several epochs by integer rounding. On the other hand, if the elevation is lower than 30 degrees, the tropospheric zenith delay must be estimated with ambiguities. The proposed algorithm estimates ambiguities and tropospheric zenith delay simultaneously utilizing ambiguity free observations of high elevation satellites. Ambiguities for high elevation satellites are resolved by integer rounding in several epochs. The algorithm has been verified by generating the simulated observation data for the 'Cheon-an' and 'Boen' reference stations in the Korea.
\end{abstract}

\section{INTRODUCTION}

Network RTK is one of the carrier phase differential GPS system, which broadcasts correction messages to users for providing $\mathrm{cm}$ level precise positioning. Correction messages could be generated as soon as the station ambiguities are fixed. Therefore reference station need reduce the time to fix ambiguity.

For reducing the time to fix ambiguities, many researchers have been studied. In these days, triple frequency ambiguity resolution is one of the major topics to shorten the time to fix ambiguity. Also the modernized GNSS systems plans and already broadcasts additional frequency measurements to improve their performance. BEIDOU and GLONASS broadcasts more than 2 frequency measurements. GPS plans L5 signal full operation since 2020.[1]

Therefore many researchers have already investigated efficient coefficients. In general, two independent ambiguities, which have meter level wave lengths, could be fixed even if with integer rounding in several epochs. They are called Extra Wide Lane(EWL) and Wide Lane(WL). However, to be free from ambiguities, one more independent ambiguity must be resolved. [2]

The last ambiguity is generally called narrow lane(NL) which has about $10 \mathrm{~cm}$ wave length. Since the difficulty to overcome other errors for short wave length, many studies have been assumed the measurement errors for several baseline lengths. [1]

In addition to previous researches, we have investigated measurement errors regarding to elevation angles in [4]. The errors depend on elevation angle as well as the baseline length. Utilization of measurement errors regarding to elevation angle, the time to fix for each interval was studied. For high elevation satellites, it is possible to determine the ambiguity in several seconds. On the other hand, if elevation angle is lower than 30 degrees, the tropospheric zenith delay must be estimated with ambiguities. Therefore the paper proposed an algorithm which has different strategies for high and low elevation satellites. In this paper, the algorithm was assessed for several environments.

We test the algorithm for 'Cheon-an' and 'Boen' reference stations in the Korea which has about $70 \mathrm{~km}$ baselines and conducted in three cases. The first case is that all GPS satellites transmit triple frequency signals. Currently, however, the GPS modernization project is underway, so only BLOCK IIF satellites has triple frequencies. Therefore, a case where only a part of satellites broadcasts triple signals is also examined. Finally, a dual frequency environment is handled and compared with others.

\footnotetext{
Corresponding author: kee@snu.ac.kr
} 


\section{ALGORITHM}

\subsection{Geometry Based}

Pseudo range and carrier phase are composed of distance, satellite clock error, troposphere delay, ionosphere delay and user clock error. Carrier phase has one more component, which is ambiguity. By double differencing pseudo range and carrier measurements, clock errors are eliminated.

For reducing or eliminating measurement errors, many researchers have combined pseudo range $\left(\rho_{1}, \rho_{2}, \rho_{3}\right)$ and carrier phase measurements $\left(\phi_{1}, \phi_{2}, \phi_{3}\right)$ for three different frequencies $\left(f_{1}, f_{2}, f_{3}\right)$.

The following equation (1) is general triple frequency geometry based combination formula. The combination coefficients $(\mathrm{i}, \mathrm{j}, \mathrm{k}, \mathrm{l}, \mathrm{m}, \mathrm{n})$ are all integers.

$$
\rho_{(l, m, n)}=\frac{l \cdot f_{1} \cdot \rho_{1}+m \cdot f_{2} \cdot \rho_{2}+n \cdot f_{3} \cdot \rho_{3}}{l \cdot f_{1}+m \cdot f_{2}+n \cdot f_{3}} \quad \phi_{(i, j, k)}=\frac{i \cdot f_{1} \cdot \phi_{1}+j \cdot f_{2} \cdot \phi_{2}+k \cdot f_{3} \cdot \phi_{3}}{i \cdot f_{1}+j \cdot f_{2}+k \cdot f_{3}}
$$

Eq. (2) shows the triple frequency combination for Double Differenced (DD) measurements. The effect of L1 ionosphere delay could be expressed with $\beta_{(i, j, k)}$. $\varepsilon_{\Delta \nabla \rho_{(l, m, n)}}, \varepsilon_{\Delta \nabla \phi_{(i, j, k)}}$ are noise for DD pseudo range and DDCP for the combination. $\lambda_{(i, j, k)}$ is the ambiguity wave length.[5]

$$
\begin{aligned}
& \Delta \nabla \rho_{(l, m, n)}=\Delta \nabla d+\Delta \nabla T+\beta_{(l, m, n)} \Delta \nabla I_{1}+\varepsilon_{\Delta \nabla \rho_{(l, m, n)}} \\
& \Delta \nabla \phi_{(i, j, k)}=\Delta \nabla d+\Delta \nabla T-\beta_{(i, j, k)} \Delta \nabla I_{1}-\lambda_{(i, j, k)} \Delta \nabla N_{(i, j, k)}+\varepsilon_{\Delta \nabla \phi_{(i, j, k)}}
\end{aligned}
$$

In this paper, geometry based measurement, which was eliminated distance and troposphere delay with broadcast orbits and Saastamoinen model, is applied for ambiguity resolution.

\subsection{Time to fix for each ambiguity}

In previous research, EWL is fixed in 1 epoch. Furthermore, we have studied WL and NL geometry based combinations characteristics for solar max activity duration. [4]

For WL, the integer rounding of $\Delta \nabla \phi_{(1,-6,5)}$ is $99.7 \%$. It seems that the triple frequency WL could be solved in 1 epoch and the dual frequency WL is fixed in 25 epoch.

However NL has low success rate for low elevation angle. Using the error analysis for elevation angle intervals, Table 1 could be calculated. The time to fix is the minimum necessary epochs which the integer rounding success rate exceeds $99.7 \%$. It shows that elevation higher than 30 degree could be fixed with integer rounding method without estimated troposphere delay.
Table 1 The time to fix for each elevation range

\begin{tabular}{|c|c|c|c|c|c|}
\hline \multirow{2}{*}{$\begin{array}{c}\text { Elevation } \\
\text { range }\end{array}$} & \multicolumn{5}{|c|}{ combination } \\
\cline { 2 - 6 } & $(4,-3,0)$ & $(4,-1,-2)$ & $(4,-2,-1)$ & $(154,0,-115)$ & $(154,-120,0)$ \\
\hline $85 \sim 90$ & 2 & 2 & $\mathbf{1}$ & 2 & 2 \\
\hline $80 \sim 85$ & 2 & 2 & $\mathbf{1}$ & 2 & 2 \\
\hline $75 \sim 80$ & 2 & 2 & $\mathbf{1}$ & 2 & 2 \\
\hline $70 \sim 75$ & 2 & 2 & $\mathbf{1}$ & 2 & 2 \\
\hline $65 \sim 70$ & 2 & $\mathbf{2}$ & $\mathbf{2}$ & 2 & 2 \\
\hline $60 \sim 65$ & 2 & $\mathbf{2}$ & $\mathbf{2}$ & 2 & 2 \\
\hline $55 \sim 60$ & 2 & $\mathbf{2}$ & $\mathbf{2}$ & 2 & 2 \\
\hline $45 \sim 55$ & 2 & $\mathbf{2}$ & $\mathbf{2}$ & 2 & 3 \\
\hline $40 \sim 45$ & 3 & $\mathbf{2}$ & $\mathbf{2}$ & 2 & 3 \\
\hline $35 \sim 40$ & 5 & $\mathbf{4}$ & $\mathbf{4}$ & 5 & 7 \\
\hline $30 \sim 35$ & 25 & $\mathbf{1 6}$ & 17 & 19 & 33 \\
\hline
\end{tabular}

\subsection{Algorithm for low elevation satellites}

Using Table 1, high elevation satellites could be resolved in several epochs. On the other hand the low elevation satellites suffer from troposphere delay due to short wave length.

Eq. (3), (4) are high elevation and low elevation measurements. For low elevation satellite ambituity resolution, ionofree measurement is selected to estimate troposphere delay. High elevation measurement could be free from ambiguity by integer rounding in several epochs. Therefore the ambiguity solved measurement would be helpful to estimate zenith troposphere delay.

$$
\begin{aligned}
z_{\text {high }} & =\left(\Delta \nabla \phi_{(77,-12,46)}-\Delta \nabla d_{b r d c}-\Delta \nabla T_{\text {model }}-\Delta \nabla N_{(77,-12,46)} \cdot \lambda_{(77,-12,46)}\right) \\
& =\Delta \nabla \delta d+\Delta \nabla \delta T+\Delta \nabla \varepsilon_{\phi_{(77,-12,46)}} \\
z_{\text {low }} & =\left(\Delta \nabla \phi_{(77,-12,-46)}-\Delta \nabla d_{\text {brdc }}-\Delta \nabla T_{\text {model }}\right)-\frac{\left(77 \cdot \Delta \nabla N_{W L}-46 \cdot \Delta \nabla N_{E W L}\right)}{(77-46)} \cdot \lambda \\
& =\Delta \nabla \delta d+\Delta \nabla \delta T+\Delta \nabla N_{(0,1,0)} \cdot \lambda+\Delta \nabla \varepsilon_{\phi_{(77,-12,-46)}}
\end{aligned}
$$

Eq. (5) is the measurement equation for low elevation ambiguities and zenith tropo delay of two stations.

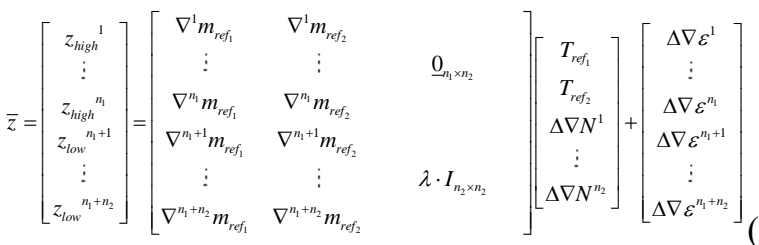

\section{SIMULATION}

The algorithm has been assessed by generating the simulated observation data for the 'Cheon-an' and 'Boen' reference stations in the Korea which has about $70 \mathrm{~km}$ baselines. It is on September $1^{\text {st }}, 2014$ because the day was one of solar max activity.

Simulated data was generated using NGA precise orbits of antenna phase center, zenith tropo delay by PPP results, Neill mapping function, and IONEX data.

Three cases were conducted in three cases. CASE 1 is that all triple frequency signals are available. CASE 2 is only 
BLOCK IIF triple frequency available situation and CASE 3 is dual frequency case.

The initial state of zenith tropo delay error is zero and the initial standard deviation is $30 \mathrm{~cm}$. The initial ambiguity for each satellite is residual of ionosphere measurement eliminated distance and troposphere delay using broadcast orbit and Saastamoinen model.

\subsection{Without integer rounding for high elevation}

For ionofree measurements, troposphere error should be estimated to solve the ambiguity. Fig 1 shows the NL ambiguity error and station differenced zenith troposphere delay error. The green line is plus 3 sigma for states. Table 2 show the time to fix for each satellite. It was high order sorted for mean elevation. PRN 16 is fixed in 2 seconds for all cases because the troposphere delay affects nearly zeros.

For triple frequency measurements, EWL and WL ambiguities are resolved in only 2 epochs. However dual frequency WL is fixed in 26 epochs. Therefore, the NL ambiguity of dual frequency satellites, CASE 2 except block IIF and CASE 3, could be estimated after 26 epochs. In figure 1, station differenced zenith troposphere delay error of case 3 is most fast to be estimated.

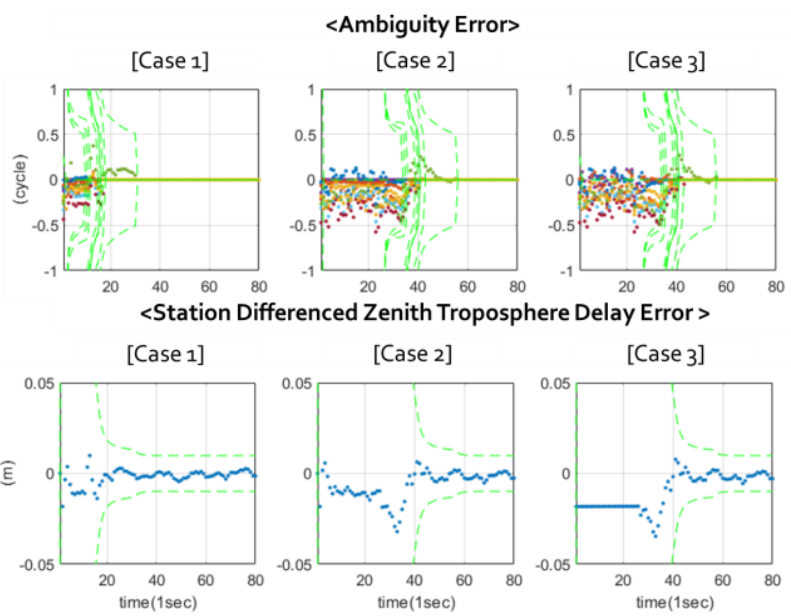

Figure 1 Ambiguity and station differenced zenith tropo sphere delay error without integer rounding for high elevation

Block IIF satellites are PRN 1, 8, 30 for this simulation. Therefore, case 1 shorten almost 20 epoch compared to case 2 and case 3 . Because the dual frequency ambiguity estimation starts after 26 epochs, sum of EWL and WL ambiguity fixing time. As the result, by reducing WL ambiguity time to fix, the ambiguity resolution has shorten in table 2 and 3.
Table 2 The time to fix and elevation without integer rounding for high elevation

\begin{tabular}{|c|c|c|c|c|}
\hline \multirow{2}{*}{ PRN } & \multirow{2}{*}{ elevation } & \multicolumn{4}{|c|}{ time to fix } \\
\cline { 3 - 5 } & & case 1 & case 2 & case 3 \\
\hline 11 & 69 & 2 & 2 & 2 \\
\hline 4 & 48 & 10 & 34 & 34 \\
\hline 28 & 46 & 11 & 35 & 35 \\
\hline 30 & 41 & 12 & 36 & 36 \\
\hline 8 & 32 & 15 & 39 & 39 \\
\hline 32 & 31 & 17 & 41 & 41 \\
\hline 19 & 29 & 16 & 41 & 41 \\
\hline 7 & 29 & 16 & 40 & 40 \\
\hline 20 & 24 & 18 & 43 & 43 \\
\hline 17 & 18 & 31 & 56 & 56 \\
\hline
\end{tabular}

\subsection{The application of integer rounding for high elevation}

Figure 2 and table 3 are the results of the proposed algorithm. Table 3 is 6-32 epoch faster than Table 2. The integer rounding for high elevation satellites helps troposphere delay error estimation and it results other ambiguity resolution to be fastened.

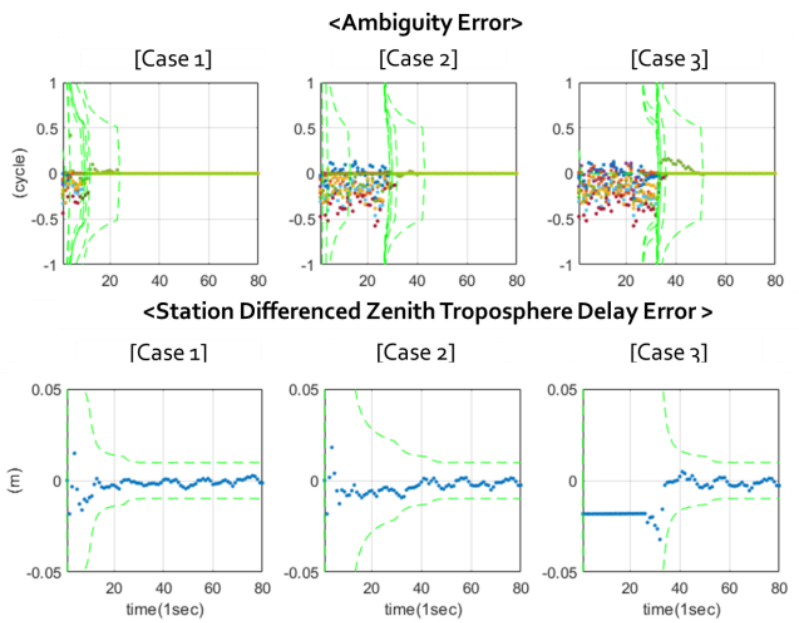

Figure 2 Ambiguity and station differenced zenith tropo sphere delay error without integer rounding for high elevation 
Table 3 The time to fix and elevation with integer rounding for high elevation

\begin{tabular}{|c|c|c|c|c|}
\hline \multirow{2}{*}{ PRN } & \multirow{2}{*}{ elevation } & \multicolumn{3}{|c|}{ time to fix } \\
\cline { 3 - 5 } & & case 1 & case 2 & case 3 \\
\hline 11 & 69 & 2 & 2 & 2 \\
\hline 4 & 48 & 4 & 29 & 33 \\
\hline 28 & 46 & 4 & 29 & 33 \\
\hline 30 & 41 & 4 & 4 & 33 \\
\hline 8 & 32 & 9 & 13 & 33 \\
\hline 32 & 31 & 11 & 30 & 33 \\
\hline 19 & 29 & 10 & 30 & 34 \\
\hline 7 & 29 & 10 & 29 & 34 \\
\hline 20 & 24 & 12 & 32 & 36 \\
\hline 17 & 18 & 24 & 43 & 51 \\
\hline
\end{tabular}

\section{Conclusion}

The proposed triple frequency ambiguity resolution algorithm, which uses integer rounding method for high elevation satellites, has been tested by simulation in three cases. Three cases are classified with the number of triple frequency broadcasting satellites. For WL ambiguity, triple frequency ambiguity resolution is 24 epochs faster than dual frequency satellites. With the integer rounding method, the time to fix for NL was shortened 6 to 32 epochs. The integer rounding for high elevation satellites helps low elevation satellite ambiguity resolution as well as zenith troposphere delay estimation.

\section{Acknowledgement}

This research was supported by a grant (18TLRPC113269-03) from Transportation logistics research Program funded by Ministry of Land, Infrastructure and Transport of Korea government. This research was supported (in part) by the Institute of Advanced Aerospace Technology at Seoul National University. The Institute of Engineering Research at Seoul National University provided research facilities for this work.

\section{References}

1. B. Li, The Journal of Global Positioning Systems, 16, 1 (2018).

2. X. Wang, W. Liu, G. Sun, Journal of Navigation, 69, 1393-1408.(2016).

3. B. Li, Y. Feng, GPS solutions, 14, 177-184 (2010).
4. S. Yu, D. Kim, J. Song, C. Kee, IPNT, (2018)

5. Y. Feng, C. Rizos, GPS Solutions, 13, 43-56 (2009). 